\title{
Architecture des gloses dans la première traduction en français de La Cité de Dieu (avant 1380)
}

\author{
Marta Andronache \\ ATILF CNRS Nancy Université \\ marta.andronache@atilf.fr
}

\section{Introduction}

Nous nous proposons d'aborder la première traduction de La Cité de Dieu en français effectuée par Raoul de Presles, sous l'angle de la structure sémantico-syntaxique et lexicale de la construction du discours. Avocat du roi, siégeant au Parlement et faisant partie des douze conseillers lais de la cour de Charles V qui le charge de traduire le De civitate Dei de saint Augustin, traduction qu'il achève certainement avant 1380. Au moment où il se voit attribuer la grande charge et lourde tâche de traduire le texte augustinien, Raoul de Presles était assez âgé et il avait déjà traduit le Compendium morale de re publica, sur l'ordre de l'évêque de Chartres, Jean d'Auvergnat et la Musa, destinée à Charles V (Bossuat 1974: 154). La traduction de La Cité de Dieu, tout comme celles qu'il avait réalisées auparavant, est une œuvre destinée à la vulgarisation du texte latin savant en français, écrit dépourvu d'originalité puisqu'il s'agit d'une traduction glosée dont les commentaires sont, pour la plupart, repris à ses prédécesseurs, Thomas Wallensis et Nicolas Trivet (Vallet 1913 : 100). Pourtant la traduction de La Cité de Dieu par Raoul de Presles a connu un immense succès à son époque mais aussi devant la postérité, puisque nous avons une très riche tradition manuscrite : plus de 50 manuscrits qui nous ont été transmis jusqu'à aujourd'hui (Laborde 1909).

Le but de notre contribution est de mettre en évidence la nouveauté que Raoul de Presles apporte sur le plan de la construction de son discours, qu'il a réussi à adapter du point de vue du style et du message au public auquel il s'adressait. Les gloses et les reprises paraphrastiques qu'il intègre à sa traduction de $L a$ Cité de Dieu contiennent l'amorce d'un discours argumentatif en français, ce qui se faisait traditionnellement en latin auparavant, et qui engendre des changements importants au niveau sémantique, syntaxique et lexical. Cette réflexion nous amène à aborder de manière succincte l'analyse des modifications que Raoul de Presles fait intervenir au niveau de la macrostructure du discours, ce qui ouvre aux linguistes un large champ d'études.

\section{Problématique}

L'œuvre de Raoul de Presles s'inscrit dans un mouvement de traductions glosées (Bertrand 2002 : 111 ; 2004 : 46-47 ; Duval 2007 : 23-24) qui s'affirme dans la seconde moitié du $14^{\mathrm{e}}$ siècle. A cette époque, les traductions non littéraires du latin commencent à être accompagnées par des gloses rédigées directement en langue vernaculaire. C'est le cas de la traduction du Policratique de Jean de Salisbury par Denis Foulechat, œuvre achevée en 1372 et effectuée à la demande de Charles V (Brucker 1969; Bertrand 2003 et 2004), ou encore des traductions commentées qu'Évrart de Conty, médecin de Charles V, fait à la même époque (Goyens 2004 ; Guichard-Tesson 2006). Nous comprenons par la glose, non pas seulement une annotation brève destinée à expliquer le sens d'un mot difficile ou d'un passage obscure, mais aussi le commentaire explicatif ou critique accompagnant la traduction.

Denis Foulechat introduit déjà des gloses à l'intérieur des chapitres sous forme de courtes explications sur le sens du texte ou d'un mot, comme patricide par exemple (Policratique 1994 : 99). La technique de la 
traduction de La Cité de Dieu est encore différente puisqu'elle est orientée plus volontiers vers le sens que vers le mot. Raoul de Presles va encore plus loin et, en traduisant La Cité de Dieu, il intègre ses gloses à son texte en deux temps : tout d'abord, à l'intérieur même du texte traduit et, par la suite, dans ses « expositions » qui accompagnent presque chaque chapitre. Les « expositions » représentent des gloses qui reprennent et mettent en discussion les principaux sujets atteints dans la traduction proprement dite. Raoul de Presles intègre ainsi son propre discours dans le texte de la traduction de La Cité de Dieu.

À travers le contenu des explications qu'il donne, le traducteur se fait l'écho de son lectorat et ce n'est alors plus surprenant que les «expositions» de Raoul de Presles soient souvent plus longues que les chapitres eux-mêmes. Le rôle des gloses est d'expliquer les sens du texte traduit et de lancer des pistes de lectures par des commentaires, digressions et anecdotes sur son temps. Du point de vue lexical, ces gloses représentent les parties les plus originales d'un ouvrage qui ne devait pas l'être, puisqu'il s'agit d'une traduction. À l'intérieur de ce discours glosé, le traducteur crée des néologismes formels ou nouveaux lexèmes, des néologismes sémantiques parfois en créant des nouveaux signifiants, comme c'est le cas de corruption-corrompre (Bertrand 2002), et des fois en créant une nouvelle acception d'un vocable.

Dans le passage de la traduction aux gloses s'affirme un discours argumentatif original. La technique de traduction de Raoul de Presles marque une nouveauté dans la pratique des traductions au $14^{\mathrm{e}}$ siècle, ce dont il a conscience puisqu'il l'invoque dans la Dédicace qui précède sa traduction :

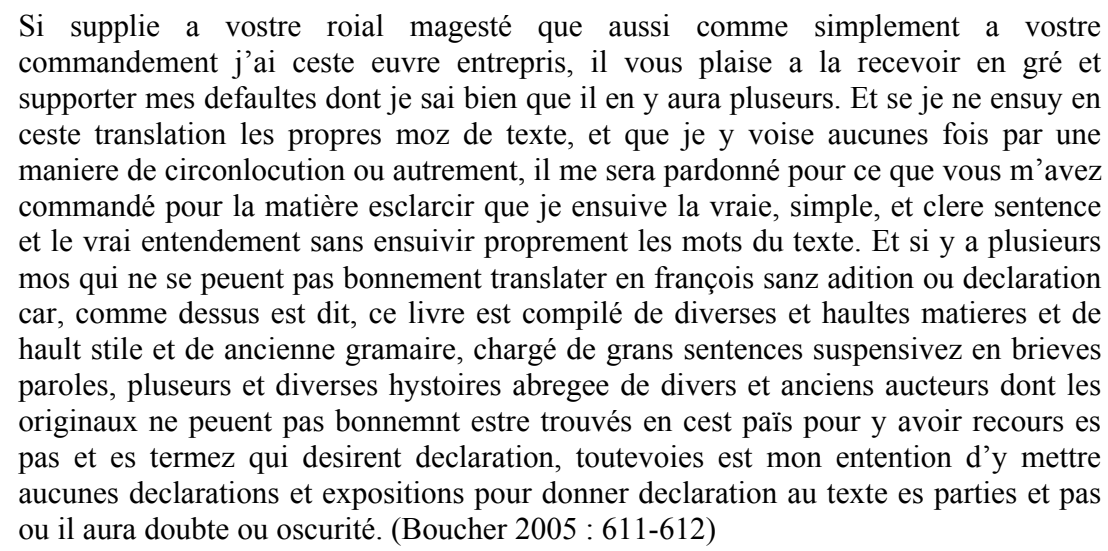

Le traducteur affirme ici clairement les principes de la technique de traduction qu'il entend appliquer dans son ouvrage. Ce passage nous montre aussi que Raoul de Presles est conscient de mettre en pratique un procédé nouveau, qui aurait pu lui attirer des critiques, mais qui répond à la demande de Charles $\mathrm{V}$ et qui fait finalement son succès.

Le roi avait commandité cet ouvrage dans le cadre d'une politique de vulgarisation des textes savants non littéraires auprès d'une haute société qui ne lisait plus aisément le latin classique, les illiterati. Le traducteur est conscient du fait qu'on ne lui demandait pas une ouvre originale du point de vue de sa matière, mais que son effort devait se concentrer surtout au niveau de la compréhension de sa traduction par son lectorat. Dans ce souci de clarté, Raoul de Presles adapte le discours à son public et il accompagne presque chaque chapitre par des «expositions » qui reprennent et expliquent le texte de la traduction là où il pouvait y avoir « doubte ou oscurité ». En effet, le fait même d'introduire sa traduction par un appareil critique et par des commentaires techniques, comme la Dédicace, le Prologue au lecteur et même un Prologue de la seconde partie, témoigne de la volonté de Raoul de Presles d'argumenter sa manière de traduire et d'expliquer la structure de son discours avec ses «defaultes » par rapport à la tradition et avec son originalité. Dans cet effort d'intégrer son propre discours argumentatif dans le texte 
même de la traduction s'affirme une langue vernaculaire savante plus spontanée qui s'allège dans son expression par rapport à la langue des traductions du latin, et qui gagne en souplesse et en qualités communicatives auprès de son public. Nous nous proposons désormais de montrer quelques changements sémantiques, syntaxiques et lexicaux dus à une émancipation de l'emprise du latin à travers les gloses et les reprises paraphrastiques.

\section{Architecture sémantico-syntaxique}

Raoul de Presles modifie la représentation du texte latin de base par un système élaboré de gloses et de paraphrases. Il glose surtout dans un espace discursif spécifique qu'il appelle "expositions », mais il produit déjà des paraphrases à l'intérieur même de la traduction. Il s'agit quelquefois de reprendre simplement un terme ou une situation, de la reformuler et de l'expliquer, comme dans cet exemple sur le « droit naturel»: « quant il parle du droit de Venus quant à la matiere presente, il le prent pour le droit naturel qui est commun à toute femme, c'est assavoir le corps du mari aussi comme au contraire, le droit du mari est le corps de la femme » (La Cité de Dieu, ms BnF fr. 22912 : 3, 3). Parfois, ses paraphrases ne sont pas exactes et elles servent au traducteur à établir une relation d'équivalence syntaxique à l'intérieur de laquelle il ajoute de l'information. La production des paraphrases se fait par des règles lexicales de type substitution synonymique et par des règles sémantiques d'équivalences approximatives obtenues par des ajouts sémantiques, communicatifs et stylistiques. Le but de Raoul de Presles n'est pas de donner une traduction fidèle du texte latin. Il opte pour une technique qui implique créativité et répétitivité recherchées. Dans la macrostructure du texte, le traducteur ajoute un préambule, une introduction et des « expositions » par rapport au texte de base, il reprend et reformule le texte pour donner des pistes de lecture, pour expliquer la traduction quant à son lexique et à ses sens et, finalement, pour influencer la réception du texte de saint Augustin par son lectorat.

Les ajouts sémantiques, communicationnels et informationnels que Raoul de Presles introduit correspondent à une situation de communication dont il faut bien déterminer les particularités : le texte de La Cité de Dieu a été écrit en latin au $5^{\mathrm{e}}$ siècle par un des pères de l'Église chrétienne, tandis que la traduction elle-même a été commanditée par le roi de France, Charles V, et elle a été effectuée par Raoul de Presles, avocat à la cour du roi à la fin du $14^{\mathrm{e}}$ siècle, pour un public français d'élite, mais principalement laïque. En prenant le risque de faire un anachronisme, nous pouvons identifier dans la façon dont le traducteur communique envers son lectorat et dans son effort d'expliquer le texte traduit, une des questions centrales de la théorie sens-texte formulée par Mel'čuk : "What information has to be added to ' $\mathrm{S}$ ' in order to ensure the production of exactly the sentences the speaker intends to produce ? » (Mel'čuk, 2001: 3). Il est évident pour nous que l'ajout d'information opéré par le traducteur médiéval par rapport au texte latin d'origine devait répondre aux attentes de son public.

Ces changements impliquent que les structures sémantiques du texte latin et de sa traduction en langue vernaculaire soient modifiées et enrichies. L'enrichissement sémantique est créé à la faveur d'une distance évidente entre l'écriture du texte latin et celle de la traduction et du commentaire qui oblige Raoul de Presles à opérer des ajouts, des gloses, des explicitations. Cet écart de la représentation sémantique de la traduction se produit, dans notre cas, par ajout d'information. Pour donner un exemple, dans le premier chapitre du Livre 3 on repère un premier niveau sémantique constitué par le texte latin qui donne : « excepto uno populo Hebraeo ... » (De Civitate Dei 1928 : 99). Dans la traduction le traducteur opère déjà des modifications et rajoute de l'information: "excepté par aventure I pueple hebrieu et aucuns autres de hors ce pueple qui furent de ce preservés par grace divine et par aucun secret et trés juste jugement de Dieu. » (La Cité de Dieu, ms BnF fr. 22912: 3, 1). Dans l'« exposition » les informations ajoutées se multiplient de manière évidente : " Aprés, quant il parle et fait exception du pueple hebreu et d'aucuns autres hors ycelli pueple qui ont este reservez, il semble qu'il se puest entendre pour une 
maniere de gent qui sont appellez bragmani ou gignosophiste ou pour les vechabites. De ces bragmanis parle monseigneur saint Gerosme ou prologue de la Bible et dit que ilz sont en Inde et que ce sont une gent qui vacquent à apprendre science et apprendre le cours des estoilles.» (La Cité de Dieu, ms BnF fr. $22912: 3$, 2). Le schéma des relations informationnelles entre segments est représentée ci-dessous (Tableau 1). On encode par $\stackrel{ \pm}{\rightarrow}$ une relation du type ajout d'information et par $\stackrel{\Rightarrow}{=}$ une relation du type explicative :

Tableau 1:

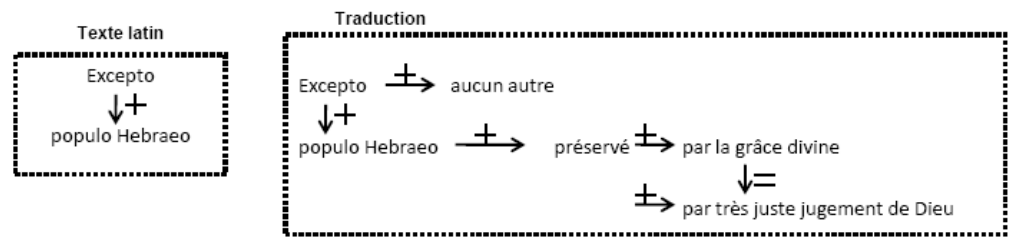

Gloses (" exposition ")

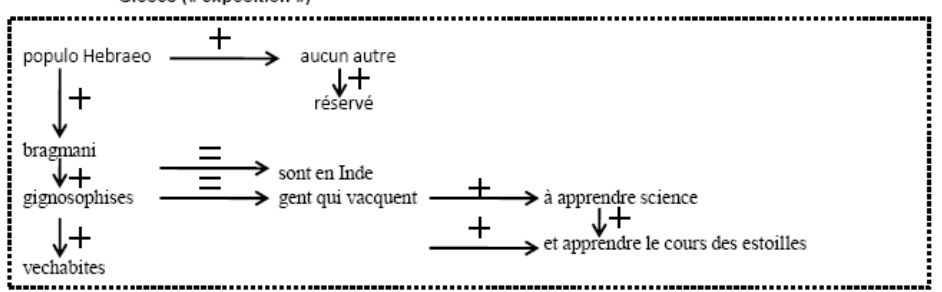

Raoul de Presles produit des paraphrases que nous pouvons repérer après avoir ôté une première strate de la structure sémantique du texte, plus précisément les ajouts informationnels, communicatifs et rhétoriques. Cela fait que la reformulation se fait en plusieurs temps : premièrement dans la traduction elle-même, puisque le texte latin n'est jamais traduit mot à mot mais qu'il est reformulé, deuxièmement, dans les « expositions » où le traducteur glose librement autour du texte de base.

La restructuration du contenu sémantique du texte de base implique des transferts sémantiques et des changements par retrait ou ajout d'information. Ce type de restructuration du texte met en jeux des lexèmes appartenant à la classe des connecteurs, il s'agit des marqueurs paraphrastiques du type par conséquent, aprés, toutevoyes, ainssi : au niveau des générateurs de la paraphrase, c'est le texte traduit qui assure la production des représentations paraphrastiques à un niveau quasi-équivalent. Tandis que la paraphrase en elle-même a trait à l'interprétation du contenu sémantique du texte traduit, les marqueurs paraphrastiques touchent uniquement à un aspect communicatif et rhétorique du sens ${ }^{1}$. Ils ne concernent donc pas le contenu sémantique proprement dit, mais ils caractérisent plutôt la façon dont ce contenu est annoncé pour introduire une explication. Le recours à la reprise paraphrastique reflète le potentiel de reformulation en moyen français, ce qui indique un stade de développement de l'expression écrite en langue vulgaire dont le style se libère peu à peu de l'emprise de la rhétorique latine par recours à des modèles de langage oral et à la création de néologismes lexicaux.

Au-delà des contraintes linguistiques spécifiques appartenant au style, à la syntaxe et au lexique, Raoul de Presles fonde aussi l'organisation communicative de sa traduction sur des données non linguistiques : un choix informationnel qu'il développe et qu'il enrichit en fonction de ses intentions, une adéquation entre le sens du texte et le but de sa traduction qui est celui de vulgariser l'œuvre de saint Augustin et, finalement, la prise en compte des attentes de son public. Cela fait que Raoul de Presles met en place un système de paraphrasage parfois virtuel, parfois reformulatif et qu'il propose un déchiffrage fondé non seulement sur l'actualisation des sens du texte de saint Augustin en fonction de l'univers culturel et de 
l'horizon d'attente de ses lecteurs, mais aussi adapté aux convictions politiques et religieuses de son commanditaire, le roi Charles V.

Dans les «expositions », la référence linguistique est la langue vulgaire, ce qui fait que l'expression linguistique est plus spontanée par rapport à la langue de la traduction, tandis que le référent sémantique est la réalité contemporaine au traducteur et non pas à l'auteur du texte. Il en découle un glissement du noyau sémantique du texte traduit vers les «expositions» qui contiennent des digressions, des explications et des citations d'œuvres faisant autorité au Moyen Âge. De manière générale, au niveau sémantique, il ne s'agit pas pour Raoul de Presles de modifier totalement le sens de la traduction quant à sa représentation, mais de l'orienter pour capter l'attention du lectorat par la reformulation du sens initial et par son adaptation aux connaissances et aux attentes de son public.

Par son procédé de doubler la traduction par des « expositions » au cours desquelles il reprend des idées du texte de base par des explications, commentaires et digressions, le traducteur produit des paraphrases à des niveaux textuels distincts, traduction et «exposition». Nous retrouvons souvent des reprises paraphrastiques et même des gloses à l'intérieur du texte traduit, mais elles sont placées surtout dans les « expositions » qui réorganisent, sans rompre sa cohérence, le message du texte initial en fonction des intentions du traducteur et des attentes de son lectorat.

Conséquemment aux changements sémantiques, la structure syntaxique est modifiée. La traduction et l'«exposition » ajoutent de l'information tout d'abord par rapport au sens du texte de base. Ensuite le traducteur change la structure syntaxique du texte traduit : ce qui était exprimé par des phrases principales dans le texte latin, est modifié en subordonnées dans l'« exposition». Les phrases de la traduction sont reformulées et le traducteur introduit des marqueurs paraphrastiques.

Au niveau syntaxique, nous constatons donc un changement dans la structure profonde des phrases initiales, puisque les phrases de la traduction sont reformulées et le traducteur introduit des lexies spécifiques et des marqueurs paraphrastiques. Les marqueurs paraphrastiques jouent le rôle de mots pivots qui introduisent différents types de paraphrases. Dans une perspective textuelle, on peut constater une articulation entre certains marqueurs paraphrastiques et le type de développement qui leur fait suite : toutevoyes et c'est a dire, par exemple, sont associés à une explication courte, être son entencion de monstrer que et par conséquant sont suivis d'un commentaire, assavoir et il semble que introduisent une digression, tandis que les structures formées avec dire peuvent introduire des gloses de tout type.

La restructuration syntaxique donne lieu à des incises qui contiennent des explications courtes sur un terme clé de la phrase : un personnage, un toponyme, une œuvre ou un fait historique et/ou littéraire. Dans ce cas, la structure du discours est la suivante: terme glosé repris par une incise explicative du type [phrase de base + c'est à dire + explication courte]. Par exemple : " Pour l'entendement de laquele chose il est assavoir que monseigneur saint Augustin veult yci mettre la fable ou ficcion qui est touchee ou livre qui s'appelle De ortu deorum, c'est à dire de la naissance des diex. » (La Cité de Dieu, ms BnF fr. $22912: 3,2)$

En ce qui suit, nous avons essayé de systématiser les marqueurs en fonction du type de subordonnée qu'ils introduisent, de leur place dans le texte, traduction ou exposition, et du type de glose qu'ils introduisent, commentaire plus développé ou simple explication. Parmi les marqueurs qui introduisent des incises, nous avons pu identifier : toutevoyes, assavoir, cest à dire, ainssi, aussi, quant à/de. Dans le tableau ci-dessous (Tableau 2), nous observons, à partir des Livres 3 et 9, leur distribution dans le texte de la traduction et des expositions, et le type de glose introduit. 


\begin{tabular}{|c|c|c|c|c|}
\hline $\begin{array}{l}\text { Marqueur de la } \\
\text { glose }\end{array}$ & $\begin{array}{c}\text { Nombre } \\
\text { d'attestations }\end{array}$ & Formes & $\begin{array}{c}\text { Traduction/Exposition } \\
\text { + explication courte }\end{array}$ & $\begin{array}{c}\text { Exposition } \\
+ \\
\text { commentaire }\end{array}$ \\
\hline toutevoyes & 10 & & oui & non \\
\hline assavoir & 30 & $\begin{array}{c}\text { c'est } \\
\text { assavoir } \\
\text { assavoir se }\end{array}$ & non & oui \\
\hline cest à dire & 50 & $\begin{array}{c}\text { c'est à dire } \\
\text { + infinitif/ } \\
\text { + nom } \\
\text { propre }\end{array}$ & oui & oui \\
\hline ainssi & 8 & et par ainssi & oui & oui \\
\hline aussi & 32 & $\begin{array}{l}\text { et aussi } \\
\text { aussi } \\
\text { comme }\end{array}$ & oui & \\
\hline quant $\grave{a} / d e$ & 48 & $\begin{array}{l}\text { quant àl } \\
\text { quant est } \\
d u l\end{array}$ & non & oui \\
\hline
\end{tabular}

Tableau 2 :

Les relatives représentent un autre type de subordonnée qui apparaît souvent dans les expositions de Raoul de Presles. Elles contiennent une explication courte du type précision sur un personnage, comme par exemple : «Didimus qui estoit leur maistre et leur evesque l'appaisa par certainnes lectres que il li envoya » (La Cité de Dieu, ms BnF fr. 22912 : 3, 1).

Les marqueurs qui introduisent des relatives sont essentiellement les pronoms relatifs, surtout qui et lequel en co-occurrence avec le verbe être. Ils sont employés à la fois dans la traduction et dans les expositions et ils peuvent introduire des explications simples aussi bien que des commentaires plus développés puisque le rôle des complétives introduites par le traducteur est de donner des explications ou des commentaires qui aident le lecteur à déchiffrer le sens du texte de base.

Les connecteurs formés sur dire qui introduisent des complétives tout en étant des marqueurs des paraphrases, représentent le cas le mieux exemplifié dans la traduction de Raoul de Presles. Leurs formes varient en fonction du contexte dans lequel ils apparaissent, traduction ou exposition, et en fonction du type de paraphrase ou de glose qu'ils introduisent : commentaire, explication, digression. Dans le tableau ci-dessous (Tableau 3), nous avons rassemblé les attestations des Livres 3 et 9 en fonction des mêmes paramètres appliqués déjà aux incises, c'est à dire la distribution dans le texte et le type de paraphrase introduite :

\begin{tabular}{|c|c|c|c|c|c|c|c|}
\hline & & & & & \multicolumn{3}{|c|}{ Attesté dans } \\
\hline $\begin{array}{l}\text { Marqueur } \\
\text { glose }\end{array}$ & & $\begin{array}{l}\text { Jombre } \\
\text { estations }\end{array}$ & Formes & & Traduction & $\begin{array}{c}\text { Exposition } \\
\text { +commentaire } \\
\text { développé }\end{array}$ & $\begin{array}{l}\text { Exposition+ } \\
\text { explication } \\
\text { courte }\end{array}$ \\
\hline dire & 84 & $\begin{array}{l}\text { Formes } \\
\text { marquées } \\
(52)\end{array}$ & $\begin{array}{c}\text { et dit que/ qui } \\
\text { dit que (20) } \\
\text { si comme dit } \\
\text { selon ce que } \\
\text { dit } \\
\text { ilz dient que } \\
\text { il dient } \\
\text { il dist que }\end{array}$ & $\begin{array}{c}+ \\
\text { complétive }\end{array}$ & oui & oui & oui \\
\hline
\end{tabular}




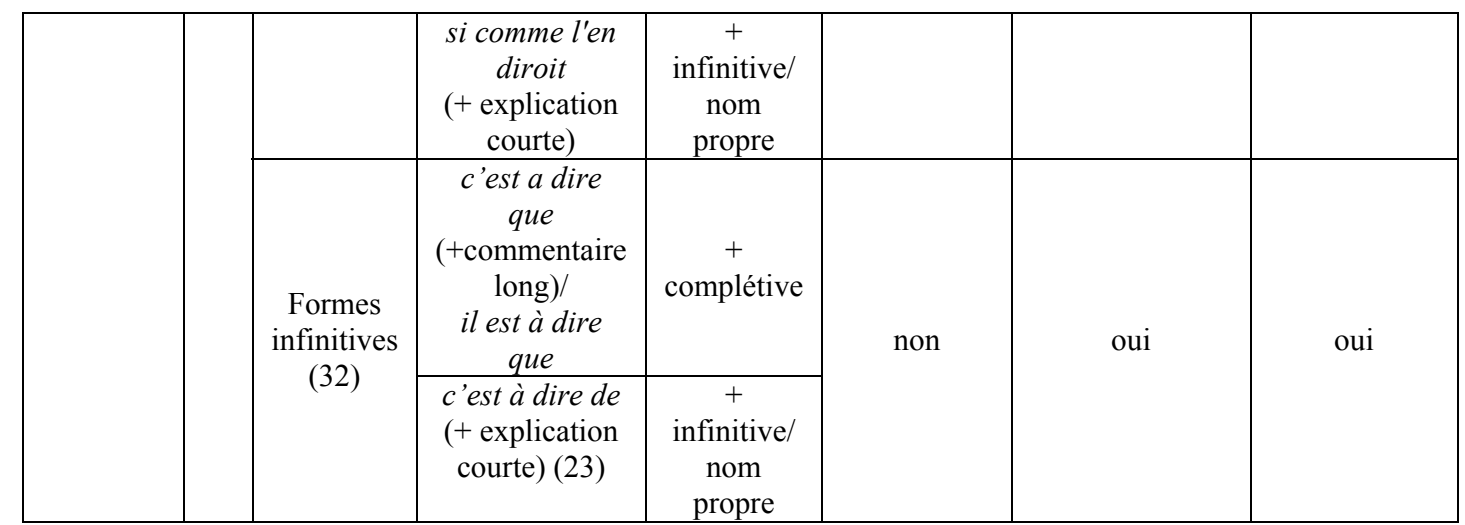

Tableau 3 :

Du point de vue textuel, on se rend compte que chacun de ces types de phrases introduit des gloses ou paraphrases spécifiques. Dans les relatives, on retrouve surtout des explications courtes : des précisions sur les personnages, sur des références littéraires, historiques ou géographiques, comme par exemple cette spécification sur la descendance de Janus : «I sien nepveu appellé samblabement Janus, lequel estoit filz de Japhet, [...]», ou bien sur les hautes fonctions publiques de Marius : « Marius lequel avoit esté VI fois consul ».

Les incises et les complétives introduisent des explications, comme par exemple cette précision sur le sens des bestes privees : « toutes les bestes privees de Romme et qui estoient subjettes à l'usage humain, c'est à dire qui habitoient entre les gens, comme chiens, chevaux, vaches, buefs et toutes autres bestes subjectés aus homes » (La Cité de Dieu, ms BnF fr. 22912 : 3, 23); ou sur le sens de parricide: «Du parricide de Romulus, c'est à dire que il tua son frere » (La Cité de Dieu, ms BnF fr. 22912 : 3, 1). Dans les incises et dans les complétives on peut trouver aussi de courts commentaires, comme dans cet exemple sur les débuts de Rome : «En ce second chapitre monseigneur saint Augustin en commence son traittié et commence à parler des maulx de painne que souffrirent les Rommains depuis leur naissance, c'est assavoir depuis que Troye fu destruite et que Enee s'en vint par mer fuiant et arriva en Ytalie. » (La Cité de Dieu, ms BnF fr. 22912 : 3, 2)

Nous avons le même type de traitement d'information dans le cas des complétives, comme dans cet exemple où le traducteur donne des explications sur la vie des brahmanes : «De ces bragmanis parle monseigneur saint Gerosme ou prologue de la Bible. Et dit que ilz sont en Inde et que ce sont une gent qui vacquent à apprendre science et apprendre le cours des estoilles. » (La Cité de Dieu, ms BnF fr. $22912: 3,1)$.

Les restructurations syntaxiques opérées par Raoul de Presles sont destinées à des fins pragmatiques, et les subordonnées introduites par le traducteur servent à donner des explications et à lancer des pistes de lecture. En même temps, ces changements par rapport à la structure grammaticale du texte de base produisent aussi des effets stylistiques puisque l'ajout d'un verbe, par exemple, permet à la langue de gagner une plus grande souplesse syntaxique.

\section{Architecture lexicale des gloses chez Raoul de Presles}

$\mathrm{Au}$ niveau du développement textuel, nous retrouvons chez Raoul de Presles trois types de gloses identifiées par Frédéric Duval (Duval 2007 : 304-305) comme éléments essentiels des « expositions » et 
de la traduction en général : l'identification des personnages et des toponymes, la définition des termes techniques, le commentaire des allusions littéraires ou historiques. A partir de ces éléments, nous avons identifié deux problématiques lexicales que nous nous proposons d'illustrer dans ce qui suit : d'une part, le fonctionnement du lexique par référence au monde grec et, d'autre part, l'évolution de l'emploi des latinismes face au processus de vulgarisation de la langue dans la traduction de La Cité de Dieu par Raoul de Presles.

\subsection{Lexique faisant référence au monde grec}

Il est bien connu que La Cité de Dieu comprend des nombreuses allusions à l'antiquité romaine ( $c f$. Duval 2008, Bertrand 2008). «Fantasmé[e]s plus que connus» (Doudet 2005: 176), les figures de la mythologie grecque sont omniprésentes dans la traduction de La Cité de Dieu. Raoul de Presles ne connaissait pas le grec (Vallet 1913), ce qui fait que ces références restent de seconde main, comme pour beaucoup de ses contemporains. Car il est connu que «les $\mathrm{XIV}^{\mathrm{e}}$ et $\mathrm{XV}^{\mathrm{e}}$ siècles en France sont dominés par le modèle de la Grèce antique, hérité de la translation du Moyen Âge classique » (Doudet 2005 : 176). Parmi les références au monde grec nous avons pu en identifier quatre types dans La Cité de Dieu: mythologiques, historiques, littéraires et géographiques. Leur présence est, en général, dictée par le contenu du texte d'origine. Néanmoins, le lecteur de la traduction est surpris par les nombreuses explications et digressions que Raoul de Presles a rajoutées par rapport au texte de saint Augustin. C'est le cas, par exemple, des références au monde oriental, à Alexandre le Grand et aux brahmanes, dans l'« exposition» du premier chapitre du Livre 3. En ce qui suit, nous aborderons les marqueurs qui introduisent ces gloses, ensuite, nous déterminerons la structure des gloses pour, finalement, voir pourquoi Raoul de Presles choisit de doubler les références au monde grec par des renvois à des ouvrages qui faisaient autorité au Moyen Âge.

Les marqueurs par lesquels le traducteur introduit ses gloses autour du monde grec, renvoient principalement à une connaissance acquise indirectement, par l'intermédiaire d'ouvrages latins ou médiévaux et non pas par la lecture des auteurs grecs. Les explications portent surtout sur des faits historiques, comme dans cet exemple : «C'est assavoir depuis que Troye fu destruite et que Enee s'en vint par mer fuiant et arriva en Ytalie » (La Cité de Dieu, ms BnF fr. 22912 : 3, « exposition » du chapitre 2) ; des faits littéraires : «Et dit monseigneur saint Augustin que ces vers ne sont pas seulement de Virgile mais sont de Omer qui fu un grant poette, ce qu'il se treuvent en ces livres » (La Cité de Dieu, ms BnF fr. $22912: 3$, « exposition» du chapitre 3); et, finalement, des faits géographiques : « [...] toutevoyes est il assavoir que Troie estoit province ou region, si comme l'en diroit France ou Picardie [...]» (La Cité de Dieu, ms BnF fr. 22912 : 3, « exposition » du chapitre 2).

Dans le tableau ci-dessous (Tableau 4), nous avons synthétisé, à partir des Livres 3 et 9 de La Cité de $\mathrm{Dieu}$, les marqueurs qui introduisent les références grecques.

\begin{tabular}{|c|c|c|c|c|}
\hline \multicolumn{2}{|c|}{ Marqueur } & Type de glose & \multicolumn{2}{|c|}{ Référence } \\
\hline c'est assavoir & est il assavoir & \multirow{4}{*}{ explication } & historique/littéraire2 & géographique \\
\hline $\begin{array}{c}\text { (car/et) il dit } \\
\text { que }\end{array}$ & cest à dire & & \multirow{3}{*}{\multicolumn{2}{|c|}{ historique/littéraire }} \\
\hline (qui) selon & iteur] + être & & & \\
\hline \multicolumn{2}{|c|}{ toutevoyes } & & & \\
\hline \multicolumn{2}{|c|}{ laquele fable est tele } & digression & \multicolumn{2}{|c|}{ historique/littéraire } \\
\hline
\end{tabular}

Tableau 4 : 
Les digressions sont introduites par des marqueurs paraphrastiques spécifiques, comme, par exemple dans l'« exposition » du deuxième chapitre du Livre $3:$ : laquele fable est tele » qui annonce une intervention plus longue de la part du traducteur au cours de laquelle il explique des faits de la mythologie grecque en exposant le sujet d'un ouvrage latin, De ortu deorum, cité par saint Augustin : " Pour l'entendement de laquele chose il est assavoir que monseigneur saint Augustin veult yci mettre la fable ou ficcion qui est touchee ou livre qui s'appelle De ortu deorum, c'est à dire de la naissance des diex. Laquele fable est tele : Laomedon qui fu roy de Troyes fist pact et convenance avec Neptunus qui est faint dieu de la mer, et avecques Apolo qui est dieu de divination, que se ilz li vouloyent ediffier la cité de Troyes, il leur paieroit tres bon loyer. » [...] (La Cité de Dieu, ms BnF fr. $22912: 3,2$ ).

Dans la structure de cette digression nous observons une construction à plusieurs paliers : référence, traduction, fable. Chacun de ces niveaux est introduit par des marqueurs paraphrastiques spécifiques : $i l$ est assavoir, c'est à dire et laquele fable est tele. Tout d'abord, nous avons une explication introduite par il est assavoir par laquelle il identifie la référence de saint Augustin ; ensuite, la traduction en français du titre est engagée par c'est à dire; et, finalement, la narration est introduite par le marqueur laquele fable est tele suivi du contenu de la fable. Au début de ce paragraphe, nous retrouvons la formule pour l'entendement de laquele chose qui fonctionne comme un marqueur-annonceur pour la digression dans son ensemble. Nous constatons qu'il s'agit de gloses qui se construisent par addition d'information et que Raoul de Presles multiplie ses explications autour du monde grec en faisant appel à des textes latins et médiévaux.

De manière presque régulière, les gloses autour du monde grec sont accompagnées de renvois à la Bible ou à d'autres autorités reconnues au Moyen Âge, comme nous avons pu le voir dans l'exemple précédent où il invoque trois sources, mythologie grecque, ouvrage latin De ortum deorum et texte de saint Augustin, pour appuyer ses commentaires. Ainsi, il renvoie, par exemple, à la Bible et à Vincent de Beauvais pour renseigner sur la longue digression sur Alexandre et les brahmanes : « De ces bragmanis parle monseigneur saint Gerosme ou prologue de la Bible. [...] Lesqueles epistres tu pourras veoir par Vincent in Speculo Historiali es temps de Alixandre» (La Cité de Dieu, ms. BnF fr. 22912: 3, « exposition » du chapitre 1).

Nous constatons que chez Raoul de Presles toute référence grecque est de seconde main et se fait par l'intermédiaire d'un auteur latin. Cette observation rejoint celle d'Henri Vallet concernant la connaissance du grec par Raoul de Presles: "Raoul connaît seulement une très petite quantité de mots grecs ; ses renseignements sur la littérature grecque sont empruntés aux commentaires de Thomas Wallensis ou de Trivet» (Vallet 1913 : 100). Mais finalement ce n'est pas à la charge du traducteur : en traduisant un ouvrage fondamental des Pères de l'Église, il fait œuvre de vulgarisation auprès d'un public laïque. Son but est de traduire et de gloser le texte de saint Augustin pour le rendre compréhensible à son public en se reportant à des références qui leur étaient accessibles comme langue et comme consultation, puisque les livres cités devaient faire partie, pour la plupart, de la bibliothèque de Charles V. Finalement, la traduction de Raoul de Presles a du succès aussi grâce aux références à des ouvrages médiévaux dans le contexte de la deuxième moitié du $14^{\mathrm{e}}$ siècle, époque à laquelle on constate une reprise de la vitalité culturelle.

\subsection{Les latinismes face au processus de vulgarisation : le cas de corrumpu dans la traduction et les gloses de Raoul de Presles}

Dans le passage du discours de la traduction au discours argumentatif des gloses nous pouvons observer des changements lexicaux, mais aussi la coexistence de latinismes et de néologismes avec des mots appartenant au lexique plus commun. C'est le sujet de l'analyse lexicale que nous proposons dans ce qui 
suit : la coexistence dans l'« exposition » de Raoul de Presles du latinisme corrumpu avec des mots du vocabulaire commun, comme avoultre, houllier, ribaut, etc.

L'influence du texte latin sur la traduction française de Raoul de Presles de La Cité de Dieu est indéniable par la nature même de cette traduction du latin en français. Il est bien évident que Raoul de Presles ne pouvait pas échapper à son époque où le contact entre le français et le latin était encore très étroit surtout dans le domaine des lettres et le domaine savant. Si nous regardons la traduction, le texte français reste assez fidèle au texte latin traduit malgré un bon nombre de paraphrases et on constate qu'il n'y a pas un véritable écart, au niveau du lexique, vis-à-vis du texte latin. Par contre, l'influence du latin chrétien de saint Augustin présente dans la traduction elle-même, s'efface sensiblement dans les « expositions » qui l'accompagnent et qui sont directement rédigées en français par Raoul de Presles. Nous assistons à un processus de remplacement progressif des latinismes par des mots plus communs du français parlé. Nous proposons d'observer ce phénomène à travers l'exemple de la présence de corrumpu dans la traduction de $\mathrm{La}$ Cité de Dieu, en nous appuyant sur l'analyse plus complète de l'extension de sens de corrompre/corruption à partir du latin classique jusqu'au français moyen (cf. Bertrand 2002) .

L'adjectif et/ou participe passé corrumpu, tout comme le verbe corrumpre et le substantif corruption sont bien attestés dans la traduction de La Cité de Dieu. Le texte de la traduction du deuxième chapitre du Livre 3, pour donner un premier exemple, reste encore très proche du texte latin qui employait ce terme au sens de « corruption morale » : « corrupta civitaté », « corrupti in iudiciis », « corruptissimis moribus » (Dombart/Kolb, vol. I: 99). La traduction reprend le même vocable, avec le même sens d'altération et perversion morale appliquée, directement ou par métonymie, à un animé :

Et se il n'en croyent riens, ne nous mettent point audevant les parjuremens des Rommains où il se esmerveillent comment les diex hairent les parjuremens des Troyens, et amerent ceulz des Rommains, dont certes la conjuracion de Cateline, en tele si grant et si corrumpue cité comme Romme. Et es grant multitude de ceulz lesquiez la main et la langue nourrissoit par parjuremens ou de sanc civil. En queles autres choses pechoyent les senateurs par tant de foiz corrumpus en leurs jugemens des pueples ou populaires en suffrages ou en quelconques causes qui estoyent demeneez par le pueple en leurs conjuracions où ilz avoyent acostume à faire leurs predications, fois par eulz parjurant et mentant. Car à ces meurs trés corrumpus estoit gardee ceste ancienne maniere de jurer, non pas que pour doubte de la religion du serement l'en leur deffendist à fere les crismes et les maulx, mais afin que les parjuremens feussent ajoustes à leurs mauvaistiez. (La Cité de Dieu, Paris, ms. BnF fr. 22912, Livre 3, 2)

En regardant l'« exposition » où il glose ce paragraphe de la traduction, nous ne trouvons plus qu'un seul emploi de corrumpu, dans l'expression corrumpue cité, qui fonctionne en co-occurrence avec toute une suite de termes communs au Moyen Âge : gens crimineulz, avoultre, houllier, ribaut, puttier, vilain, joueur de dés, gourmant, gasteur de biens, murtrier parricide, sacrilliege, parjure, homme vicieux. Ces mots de la langue commune ont le rôle de reprendre et de compléter pour le lecteur contemporain à Raoul de Presles le champ sémantique de corrumpu:

Et se tu veulx veoir encores la conjuracion de Catheline, voy Saluste in Cathelinario qui dit que c'estoit tres legiere chose en si corrumpue cité, que ce Catheline feust avironné de toutes gens crimineulz. Car il n'i avoit ne avoultre, houllier, ribaut, puttier, vilain, joueur de dés, gourmant, gasteur de biens, murtrier parricide, sacrilliege, parjure, ne homme vicieux quelconques, qui ne feussent ses prouchains, et qu'il n'eust aliez à lui. (La Cité de Dieu, Paris, ms. BNF fr. 22912: 3, « exposition » du chapitre 2) 
Le fait que le traducteur accorde plus de place à des termes de la langue commune marque sa volonté de s'éloigner de l'emploi des latinismes pour parler à son lectorat dans une langue commune et rendre le sens du terme corrumpu encore plus complet et plus clair. Par conséquent, il nous semble évident qu'il existe une différence entre la langue de la traduction elle-même et la langue de l'« exposition » qui va dans le sens de l'affirmation d'une langue plus spontanée, émancipée de l'emprise des latinismes. Et pour mieux comprendre ce type de glissement lexical qui est typique pour la langue de la traduction de Raoul de Presles et qui définit finalement des ensembles sémantiques homogènes, nous proposons de regarder l'histoire de corrompre par rapport à celle de ses concurrents de la langue vulgaire, surtout houllier, ribaut, intéressants aussi pour leur origine, mais aussi avoultre, puttier, vilain, parjure, gasteur de biens.

Le verbe corrompre, emprunté au latin corrümpěre, est attesté depuis le $12^{\mathrm{e}}$ siècle, et non depuis le $11^{\mathrm{e}}$ siècle comme l'indique à tort le FEW 2,1233b, avec le sens de « altérer ce qui est sain, honnête » (ErecF $21=\mathrm{TL})^{4}$. Le sens de «corrompre une femme », « en faire sa maîtresse, violer, coïre » remonte au $12^{\mathrm{e}}$ siècle (RouH II 4257 = TL). Par contre, le sens de « altérer chez quelqu'un (par exemple chez les juges), par des cadeaux, l'intégrité de la conscience » que le FEW 2, 1234a relève depuis la fin du $13^{\mathrm{e}}$ siècle (BeaumCoutS $\S 1246=\mathrm{TLF})$, se rencontre depuis $c a 1174\left(\right.$ SThomasGuernW $\left.{ }^{1}=\mathrm{TLF}\right)$. Pour compléter le FEW, qui ne disposait pas à l'époque de la rédaction de cet ouvrage monumental d'une documentation très fournie pour la période du moyen français, nous nous proposons d'enrichir les données grâce aux articles corrompre et corruption du DMF 2009, articles rédigés par Robert Martin, tout en les confrontant avec les matériaux recueillis dans La Cité de Dieu. Parmi les trois sens dégagés par le DMF, «idées de rupture, de destruction ou d'agression » (A.), « idée d'altération » (B.), « idée de corruption morale » (C.), nous en avons trouvé deux dans les exemples que nous avons pu recenser dans La Cité de Dieu. Tout d'abord, l'«idée d'altération » est présente avec le sémantisme de «souiller (une femme) » (A. 5.). La première attestation relevée par le DMF pour ce sens est proche de la date de la traduction française de $L a$ Cité de Dieu puisque notre traduction est achevée, le plus probablement, en 1375, tandis que le poème de Machaut est daté depuis la même année 1375. Les deux textes se rapprochent aussi par l'aspect moralisateur du discours, puisque Le Confort d'ami, écrit pour Charles de Navarre emprisonné par son cousin Jean II, présente souvent des aspects moralisateurs : « [...] Athlente qui estoit fille d'un appellé Yperbolens, laquelle Hercules corrumpi et viola en ce lieu » (La Cité de Dieu, ms. BnF fr. 22912 : 3, 3). Le deuxième sens très bien attesté dans La Cité de Dieu comporte l'idée de corruption morale dans le sens défini par le DMF d'« altérer moralement (qqn, son âme, son cœur, ses sentiments, ses désirs, ses mœurs ..., une collectivité), pervertir » (C.1. et C.2.). La première datation de ce sens se trouve, $c f$. le DMF 2009, dans la partie commentaires du Livre de Ethique d'Aristote traduit par Nicole Oresme (OresmeEthM, $c f$. DEAF), texte daté de $c a 1370$, et qui se rapproche, par la datation et par le genre, de la traduction de Raoul de Presles.

A partir des exemples que nous avons trouvés dans la traduction de La Cité de Dieu, nous pouvons observer qu'est dit corrumpu un personnage et ses mœurs : «Aprés, Anthonius lequel fu moult depareil de Cesar en meurs et en vertus, et qui estoit soullie et corrumpu, convoita si comme il sembloit à avoir la puissance et seignourie de Cesar Auguste. » (La Cité de Dieu, ms. BnF fr. 22912 : 3, 30) ; ou bien : "Car à ces meurs trés corrumpus estoit gardee ceste ancienne maniere de jurer » (La Cité de Dieu, ms. BnF fr. 22912 : 3, 2). Corrumpu s'applique aussi à un personnage et à son jugement : «En queles autres choses pechoyent les senateurs par tant de foiz corrumpus en leurs jugemens des pueples ou populaires en suffrages ou en quelconques causes qui estoyent demeneez par le pueple en leurs conjuracions où ilz avoyent acostume à faire leurs predications, fois par eulz parjurant et mentant. » (La Cité de Dieu, ms. BnF fr. $22912: 3,2$ ).

Une cité peut être aussi corrumpue et, par métonymie, ses habitants : «Et se tu veulx veoir encores la conjuracion de Chatheline, voy Saluste in Cathelirnario qui dit que c'estoit tres legiere chose en si 
corrumpue cité, que ce Chatheline feust avironné de toutes gens crimineulz. » (La Cité de Dieu, ms. BnF fr. $22912: 3$, 2). Et, finalement, le substantif corruption apparaît dans le contexte des élections : «Aprés quant il parle des suffrages du pueple, il l'entent proprement des corruptions qui se faisoyent es elections ou es autres besongnes. » (La Cité de Dieu, ms. BnF fr. 22912 : 3, 2).

Comme nous pouvons l'observer à partir de ces exemples, l'adjectif corrumpu est toujours associé à des personnages situés en haut de la hiérarchie sociale et qui devraient servir de modèle. L'intention de l'auteur et du traducteur est ici évidemment moralisatrice: Anthonius «qui estoit soullie et corrumpu », les senateurs «par tant de foiz corrumpus en leurs jugemens », Hercule, un demi-dieu, qui « corrumpi et viola ». En même temps, nous pouvons constater une similitude d'emplois entre nos exemples et les citations du DMF 2009 où la cible de la corruption est un juge : «corrumpant le juge, (OresmeEthM : 499) ; " ung juge inique et corrompu», (Juvenal, Écrits politiques : 37) ; ou bien un lieutenant : " corrompirent le lieutenant du dit capitaine » (Juvenal, Écrits politiques : 117). La corruption se fait par argent (« corrumpuz par argent», Gerson, Sermon pour la Noël : 311), et, de manière comparable, dans La Cité de Dieu les sénateurs sont corrompus «par dons ou par faveurs» (La Cité de Dieu, ms. BnF fr. $22912: 3,2)$.

En ce qui concerne ces changements lexicaux, il est intéressant de voir les cooccurrences de corrumpu. Dans le sens de «souiller une femme», corrompre est doublé par un synonyme, violer, ce qui implique l'idée d'insistance sur la compréhension du sens employé dans ce contexte. Dans le sens de « corruption morale », corrumpu est le plus souvent associé à parjuremen, conjuracion et mauvaistiez, souille, convoitise, des termes qui expriment tous un mauvais état des mœurs sans être des synonymes. Corrompu se trouve souvent en binôme ou trinôme avec un ou plusieurs de ces termes. Les co-occurrences peuvent être très rapprochées, associées par la conjonction et : «corrumpi et viola », « souillie et corrumpu »; ou bien plus éloignées mais qui restent à l'intérieur de la même séquence sémantique et syntaxique: corrumpue/parjuremens, corrumpus/conjuration/ mauvaistiez. L'exemple de «corrompu cité » est intéressant, car il s'agit d'une métonymie pour désigner ses habitants corrompus, et qui est en cooccurrence, comme nous avons pu le voir, avec une suite de douze termes qui expriment le mauvais état des mœurs de la cité de Rome : corrumpue citélgens crimineulz/ avoultre, houllier, ribaut, puttier, vilain, joueur de dés, gourmant, gasteur de biens, murtrier parricide, sacrilliege, parjure, ne homme vicieux (La Cité de Dieu, ms. BnF fr. $22912: 3$, 2).

Parmi ces lexèmes de la langue commune employés par Raoul de Presles pour rendre plus clair le sens de corrompre à son public, deux attirent particulièrement l'attention par leur origine et par leur histoire : houllier et ribaut. Le substantif masculin houlier emprunté à l'ancien bas francique *horari au sens de «homme qui vit de la prostitution de femmes ou qui fréquente des prostituées » (DEAF H 4/5, s.v. horier $^{5}$, est attesté depuis $c a .1195$ dans un miracle dramatisé de Jehan Bodel dont le manuscrit unique date probablement de 1295 (BodelNicH). Le lexème est bien attesté en moyen français, comme le prouve la richesse des citations fournies par l'article houlier du DMF, qui nous montre clairement que le mot était très vivant au $14^{\mathrm{e}}$ siècle alors que vers la fin du $15^{\mathrm{e}}$ siècle, le mot sera employé comme terme d'injure. Les quatre attestations que nous avons trouvées au $20^{\mathrm{e}}$ siècle pour houlier dans Frantext ne peuvent pas être prises en compte pour l'histoire du lexème du Moyen Âge qui, bien attesté jusqu'au $16^{\mathrm{e}}$ siècle, est totalement sorti de l'usage par la suite, ce qui fait qu'il n'existe pas d'entrée houlier dans le TLF. Un autre cas semblable, c'est le substantif ribaut, mais il s'agit cette fois d'un lexème qui atteste une continuité d'emploi et de sens à travers le temps. Le FEW atteste ribaut au sens de "homme débauché ; méchant, vagabond (t. d'injure) » depuis le $12^{\mathrm{e}}$ siècle, emprunté de l'ancien haut allemand rîban (FEW 14, 702a). Le mot est bien attesté dans six des lexiques du DMF et les citations proviennent des textes de genres tout à fait différents, chartes, miracles, chroniques, ce qui démontre la vivacité de ce terme aux $14^{\mathrm{e}}$ et $15^{\mathrm{e}}$ siècles. Une simple recherche dans Frantext nous montre que ribaud est attesté en 
continu dans le vocabulaire français jusqu'à nos jours. Pourtant c'est un lexème qui appartient au lexique du Moyen Âge. Ce n'est pas par hasard si le TLF le donne comme « vieilli ou litt. » au sens de « homme ou femme de mauvaise vie » (II.B.), ou comme «appartenant à l'histoire du Moyen Âge»: «soldat faisant partie d'un corps de garde créé par Philippe Auguste » (I.A.1.), et « aventurier qui suivait l'armée en quête de pillages » (I.A.2.) (TLF 14, s.v. ribaud).

Au terme de cette analyse lexicale, nous souhaitons répondre à la question suivante : quels sont les sens et la raison des emplois de corrumpu et de son emploi dans la traduction de La Cité de Dieu ? Selon nous, la reprise de ce terme par des synonymes et le renforcement de son sens par ajout de mots qui complètent son champ sémantique répondent au souci du traducteur, qui revêt ici aussi le rôle de lexicographe, de donner des définitions et des paraphrases synonymiques pour des mots nouveaux ou peu compris à une époque où les dictionnaires n'existaient pas encore et les glossaires n'étaient pas forcément consultés par les illiterari. Raoul de Presles fait ainsi œuvre de vulgarisation, mais aussi œuvre de lexicologie intégrée à un nouveau discours argumentatif qu'il lance à travers ses gloses et paraphrases, sans jamais oublier le sens de sa traduction : se faire bien comprendre par ses lecteurs et produire un texte rédigé dans une langue française vivante, émancipée de l'emprise des latinismes. Malgré cela, il est évident que la traduction de La Cité de Dieu comporte un grand nombre de latinismes et nous avons pu le voir déjà dans les exemples que nous venons de citer, sauf que ces latinismes sont explicités à un double niveau : tout d'abord au niveau de leur contexte par des renvois à des autorités reconnues par le public savant du Moyen Âge, comme Cicéron, Oresme ou Vincent de Beauvais et, ensuite, au niveau lexical puisqu'ils sont accompagnés soit d'un complément sémantique (corrumpue/parjuremens, corrumpus/conjuration), soit d'un ou plusieurs mots de la langue commune qui permettent un ajout sémantique et qui paraphrasent finalement le terme central.

\section{Conclusion}

La technique de traduction de La Cité de Dieu par Raoul de Presles s'inscrit dans un mouvement d'explication et de vulgarisation des traductions du latin qui répondait à la politique culturelle de Charles V (Duval 2007). Le défi lancé au traducteur était de rendre clair à un public cultivé mais qui ne lisait pas forcément le latin, les illiterati, le texte de saint Augustin. Pour atteindre ce desiderata, le traducteur met en place une véritable architecture du discours qui fonctionne, comme nous avons pu le voir, par ajout d'information par des gloses et des paraphrases introduites à tous les niveaux du discours. Ce procédé implique des ajouts communicatifs et sémantiques, ce qui engage des changements au niveau syntaxique. Au niveau lexical, les reprises paraphrastiques opèrent des glissements du domaine savant vers l'emploi d'une langue vulgaire plus spontanée. Ce processus est bien marqué surtout dans les « expositions » où le traducteur se sent libre d'introduire des digressions qui confèrent de l'originalité à sa traduction et qui nous donnent un état de l'expression de la pensée politique et théologique en langue vernaculaire à la fin du $14^{\mathrm{e}}$ siècle.

À travers les reprises paraphrastiques, la langue de la traduction de Raoul de Presles s'affirme donc par une expression plus spontanée qui annonce le passage à une production littéraire rédigée directement en langue française qui aura lieu dans le premier quart du $15^{\mathrm{e}}$ siècle avec l'œuvre morale de Christine de Pizan ou les écrits politiques de Jean de Montreuil et d'Alain de Chartier (Duval 2007). Devant le succès de la technique de Raoul de Presles, la tendance à accompagner les traductions par des commentaires continue à s'affirmer, encouragée par Charles V. C'est le cas de la traduction de Facta et dicta memorabilia de Valère Maxime, commencée en 1375 par Simon de Hesdin et achevée par Nicolas de Gonesse en 1400-1401, traduction accompagnée de commentaires et d'exemples ajoutés par rapport au texte latin. 
La traduction de La Cité de Dieu marque une différence par rapport à d'autres ouvrages contemporains du même genre, dans lesquels les gloses en langue vernaculaire restaient encore en dehors du texte. En effet, Raoul de Presles les intègre au texte traduit et affirme ainsi l'amorce d'un discours argumentatif en langue française. Dans une traduction orientée plutôt vers la compréhension que vers la traduction mot à mot, la reprise paraphrastique dans les «expositions » n'est pas un hasard. Il s'agit à ce niveau d'un discours bien structuré, d'une rhétorique travaillée, ce qui nous conduit à constater l'existence d'une véritable architecture des gloses et des paraphrases dans la traduction de La Cité de Dieu.

\section{Bibliographie}

Augustin, Saint, La Cité de Dieu, volume I, ms BNF fr. 22912, Paris.

Augustinus, Sancti (éd. 1928), De Civitate Dei, volume I, Dombart B./Kalb A. (éd.), Teubneri.

Bertrand, O. (2008). Le traitement de l'histoire romaine dans les gloses du premier traducteur français de la Cité de Dieu (1371-1375), Texte, 43/44 « Epistémocritique », Toronto,Trinity College.

Bertrand, O. (2004). Du vocabulaire religieux à la théorie politique en France au XIV siècle. Les néologismes chez les traducteurs de Charles V (1364-1380), Paris, Connaissances et Savoirs.

Bertrand, O. (2003). Les néologismes politiques dans la première traduction française de La Cité de Dieu de saint Augustin 1375, In The Theory and Practice of Translation in the Middle Ages, (The Medieval Translator. Traduire au Moyen Âge, 8), R. Voaden, R. Tixier, T. Sanchez Roura et J. R. Rytting (éd.), Turnhout : Brepols, $39-48$.

Bertrand, O. (2002). Transferts et extensions de sens dans le vocabulaire religieux : le cas de corrompre/corruption du latin classique au moyen français, In Pratiques de Traduction au Moyen Age. Actes du Colloque de l'Université de Copenhague, 25 et 26 octobre 2002, Copenhague, Peter Andersen, 111-120.

Bossuat, R. (1974). "Raoul de Presle", in Histoire littéraire de la France, 40, 113-186.

Boucher, C. (2005). La mise en scène de la vulgarisation. Les traductions d'autorités en langue vulgaire aux XIIIe et XIVe siècles, doctorat, École pratique des hautes études, Paris.

Brucker, C. (1994). Le policratique de Jean de Salisbury (1372), livres I-III, Genève : Droz.

Brucker, C. (1969). Les néologismes de Denis Foulechat, traducteur de Charles V, d'après les trois premiers livres du Policratique, Revue de linguistique romane, 33, 317-324.

DEAFBiblEI = Möhren, F./Städtler, Th., 2002- . Dictionnaire Étymologique de l'Ancien Français. Version chantier électronique du Complément bibliographique, Heidelberg, DEAF (http://www.deaf-page.de/deaff.htm).

Doudet, E. (2005). Le miroir de Jason : la Grèce ambiguë des écrivains bourguignons au XVe siècle, In La Grèce antique sous le regard du Moyen Âge occidental, Actes de colloque, Jean Leclant et Michel Zink (éd.), Paris : Diffusion de Boccard, 175-193.

Duval, F. (2008). Sémiotique des mots de civilisation romaine en français médiéval, Tübingen/Basel, Verlag.

Duval, F. (2007). Lectures françaises de la fin du Moyen Âge. Petite anthologie commentée de succès littéraire, Genève : Droz.

Goyens, M. (2004). Évrart de Conty: traducteur, adaptateur et commentateur des Problèmes d'Aristote, "Pour acquerir honneur et pris". Mélanges de moyen français offerts à Giuseppe Di Stefano, Maria Colombo Timelli et Claudio Galderisi (éd.), Montréal : CERES, 123-135.

Guichard-Tesson, F. (2006). Évrart de Conty, poète, traducteur et commentateur, In Aristotle's "Problemata" in Different Times and Tongues, Pieter De Leemans et Michèle Goyens (éd.), Leuven : Leuven University Press (Mediaevalia Lovaniensia. Series I. Studia, 39), 145-174. 
Laborde, A. de (1909). Les manuscrits à peintures de La Cité de Dieu de Saint Augustin, Paris : Rahir.

Mel'čuk, I. (2001). Communicative Organization in Natural Language. The semantic-communicative structure of sentences, Amsterdam/Philadelphia : John Benjamins Publishing Company.

Milićević, J. (2007). La paraphrase. Modélisation de la paraphrase langagière, Bern/Berlin/Bruxelles/Frankfurt am Main/New York/Oxford/Wien : Peter Lang.

SThomasGuern $^{1}=$ 1922. La Vie de saint Thomas le martyr par Guernes de Pont-Sainte-Maxence, édité par Emmanuel Walberg, Lund, Gleerup (Skrifter utgivna av Kungl. humanistiska Vetenskapssamfundet i Lund 5). Date du texte : ca 1174. Région d'origine du texte : Ouest/Angleterre (anglo-normand).

Vallet, H. (1913). La culture classique dans Raoul de Praelles d'après sa traduction avec commentaire de La Cité de Dieu de saint Augustin, in Position des thèses, Ecole Nationale de Chartres, Paris : Librairie Alphonse Picard et fils, 99-101.

\footnotetext{
1 Nous employons la notion de sens communicatif comme «spécification des intentions communicatives du locuteur», traducteur dans notre cas, et sens rhétorique comme "spécification des intentions stylistiques du locuteur » (cf. Milićević $2007: 20)$.

2 Nous avons choisi surtout pour la mythologie l'étiquette «histoire/littérature» puisque nommer ce type de références uniquement « littéraire » peut être ambigu et peut engendrer des anachronismes.

3 L'étude d'Olivier Bertrand (Bertrand 2002) analyse le groupe corrompre/corruption du point de vue de la diachronie, tandis que la présente contribution se concentre particulièrement sur le corpus du livre 3 de La Cité de Dieu.

4 Tous les sigles des éditions de texte d'ancien français sont ceux du DEAFBiblEI ou du TLF-Étym (http://www.atilf.fr/tlf-etym/).

${ }^{5}$ Nous avons retenu l'étymologie du DEAF H 4/5, s.v. horier contre celle donnée par le FEW : houlier (attesté du $13^{\mathrm{e}}$ siècle au $16^{\mathrm{e}}$ siècle) par dissimilation de hurier, de l'ancien haut allemand huorâri, "courtier de débauche, maquereau; homme qui fréquente les femmes de mauvaise vie » (FEW 16, 266a).
} 